\title{
Radiation Protection Principles Observance in Mammography Divisions in Shiraz
}

\author{
Zahra Siavashpour ${ }^{1}$, Simin Mehdizadeh ${ }^{1}$, Afrooz Farshadi ${ }^{1}$, Milad Baradaran-Ghahfarokhi \\ 2, * \\ ${ }^{1}$ Radiation Research Center and Medical Radiation Engineering, Mechanical Engineering Department, Shiraz University, Shiraz, IR Iran \\ ${ }^{2}$ Medical Physics and Medical Engineering Department, School of Medicine, Isfahan University of Medical Sciences, Isfahan 81746, IR Iran \\ * Corresponding author: Milad Baradaran-Ghahfarokhi, Medical Physics and Medical Engineering Department, Isfahan University of Medical Sciences \\ (IUMS), Isfahan 81746, IR Iran. Tel.: +98-3117922432, Fax: +98-3116688597, E-mail: milad_bgh@yahoo.com
}

Keywords: Mammography; Radiation Protection; Radiography

\section{Dear Editor,}

Breast cancer is the second most common neoplasia and the first leading cause of cancer death among women in the world (1). Early diagnosis of breast cancer plays a critical role in reducing mortality rates and improving the patient's prognosis $(2,3)$. Mammography is an extremely useful technique for detection of breast cancer (4). An important goal in this imaging modality is to obtain the best diagnostic information by delivering the least radiation $(5,6)$.

Some protocols by International Atomic Energy Agency (IAEA) are involved in the process of optimizing the radiation used for imaging, which are related to the selection of appropriate imaging equipment, evaluation of equipment performance in the context of quality assurance programs, radiation protection principles and education of medical and technical staff on the appropriate imaging procedures and protocols $(7,8)$. Considering these protocols, it is essential to have comprehensive knowledge, attitude and practice of mammography in the divisions, emphasize on the importance of its implementation as a routine and preventive measure for early diagnosis of breast cancer $(1,4)$.

The aim of this study was to investigate the radiation protection principles observance and to evaluate the existing status of radiological practice and equipment performance according to the radiation protection protocols established by IAEA in mammography division in Shiraz. According to the best of our knowledge, there was no evidence for the same study in Iran. A questionnairebased study was carried out in 5 diagnostic mammography divisions of Shiraz namely Namazi, Faghihi, Hafez, Zeinabieh, and MRI. All of the 30 women staff were interviewed between February 2008 to March 2008. All of them were asked to participate in the study. The refusal rate to participate was very low.

The above mentioned questionnaire consisted 5 main categories. First, the audiences were asked about radiation dose measurement units. The second part was about the Annual Maximum Permissible Dose (AMPD) (5, 6), the necessary training in regards to the procedures and quality assurance and radiation programs at the beginning of the work made the third and fourth ones. The fifth was about renewing the licenses. Then the questionnaires were reviewed for information quality and legitimacy, and corrections were made as needed. After reviewing the questionnaires, statistical analysis was performed using SPSS software (Version 10) by descriptive statistics.

We found that in mammography divisions in Shiraz, $47 \%$ of the staffs had not passed necessary training in regards to the procedure nor quality assurance and radiation program at the beginning of their work ( Figure 1 ). About $80 \%$ of personnel had not their licenses renewed by attending the training classes again. Moreover, $80 \%$ of the machinery and equipment were not being regulated nor monitored as often as needed. Most of the radiation workers were aware of radiation dose measurement units and AMPD (>95\%). In addition, we found that in $80 \%$ of the divisions, there was no special radiation protection

\footnotetext{
Article type: Letter; Received: 03 Aug 2011, Revised:19 Dec 2011, Accepted:20 Dec 2011; DOI:10.5812/ircmj.2004

Please cite this paper as:

Siavashpour Z, Mehdizadeh S, Farshadi A, Baradaran-Ghahfarokhi M. Radiation Protection Principles Observance in Mammography Divisions in Shiraz. Iran Red Cres Med J. 2012;14(12):840-1. DOI: 10.5812/ircmj.2004
} 
shield used for the patients. This study showed that an adequate training of staffs in mammography divisions was required to reduce the patient's radiation dose. Implementation of radiation protection courses and education of practical issues, including radiation dose received by patients and radiation safety, during medical education programs could be an effective method to reduce the patient's dose in medical exposures.

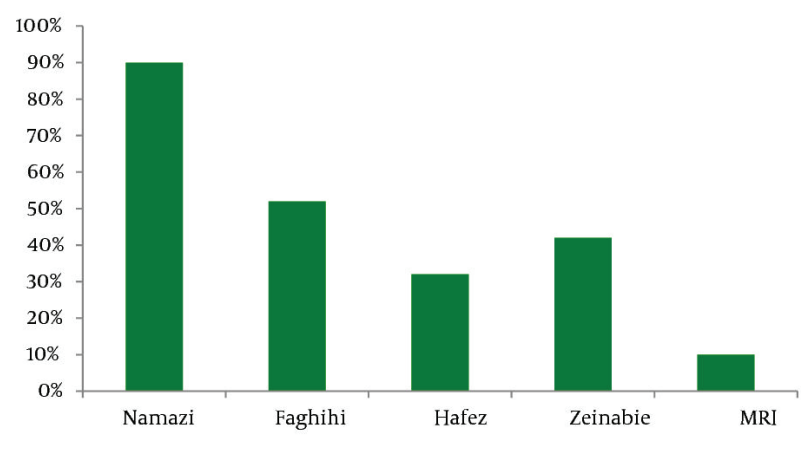

Figure 1. Status of Personnel Training in Quality Assurance and Radiation Programs

Our findings are similar to other studies in the literature $(4,9)$. In our study, the interviewed staffs were from five different divisions in Shiraz. Therefore, our results may not apply throughout Iran, but it seems that most divisions have the same problems $(4,10)$. The lack of awareness becomes particularly pertinent when we consider the number of staffs who received inappropriate training. Further investigation in other parishes of Iran is suggested. Combined to the data of the present study, both could provide better understanding of the existing status of radiological practice and equipment performance in mammography divisions.

\section{Acknowledgements}

The authors wish to acknowledge the Research Board in
Nuclear Science, Tabesh Research Center, Shiraz University, Shiraz, Iran for their critical technical support and advice.

\section{Financial Disclosure}

None declared.

\section{Funding/Support}

None.

\section{References}

1. Blanchard K, Colbert JA, Puri D, Weissman J, Moy B, Kopans DB, et al. Mammographic screening: patterns of use and estimated impact on breast carcinoma survival. Cancer. 2004;101(3):495-507.

2. Ayad M. Risk assessment of an ionizing-radiation energy in diagnostic radiology. Applied Energ. 2000;65(1):321-8.

3. Monfared AS, Abdi R, Saber M. Repeat analysis program in radiology depatments in Mazandaran province-Iran; Impact on population radiation dose. Iran J Radiat Res. 2007;5(1):237-40.

4. Marinho LA, Cecatti JG, Osis MJ, Gurgel MS. Knowledge, attitude and practice of mammography among women users of public health services. Rev Saude Publica. 2008;42(2):200-7.

5. Gray JE, American Institute of Physics, American Association of Physicists in Medicine. The Role of the Clinical Medical Physicist in Diagnostic Radiology: Description of the Role of the Clinical Medical Physicist in Diagnostic Imaging. American Institute of Physics for the American Association of Physicists in Medicine; 1994.

6. The American College of Radiology. Standards, General Radiogeraphy. 2000 [updated 2000; cited 2011]; Available from: www.acr.org.

7. International Atomic Energy Agency. Optimization of the radiological protection of patients undergoing radiography, fluoroscopy and computed tomography: final report of a coordinated research project in Africa, Asia and eastern Europe. International Atomic Energy Agency; 2005.

8. International Atomic Energy Agency. Applying radiation safety standards in diagnostic radiology and interventional procedures $u \operatorname{sing} x$ rays. International Atomic Energy Agency; 2006.

9. Jacob K, Vivian G, Steel JR. X-ray dose training: are we exposed to enough? Clin Radiol. 2004;59(10):928-34;.

10. Mubeen SM, Abbas Q, Nisar N. Knowledge about ionising and nonionising radiation among medical students. J Ayub Med Coll Abbottabad. 2008;20(1):118-21. 